\title{
H.E.S.S. data analysis with open source science tools
}

Christoph Deil ${ }^{* a}$, Michael Mayer ${ }^{b}$, Régis Terrier $^{c}$, Ignasi Reichardt ${ }^{c, g}$, Anneli Schulz $^{d}$, Axel Donath ${ }^{a}$, Johannes King ${ }^{a}$, Manuel Paz Arribas ${ }^{b}$, Stefan Ohm ${ }^{d}$, Isak Delberth Davids ${ }^{e}$ and Philipp Willmann ${ }^{f}$ for the H.E.S.S. collaboration

${ }^{a}$ MPIK, Heidelberg, Germany

${ }^{b}$ Humboldt University, Berlin, Germany

${ }^{c} A P C$, University of Paris 7, France

${ }^{d}$ DESY, Zeuthen, Germany

${ }^{e}$ North-West University, Potchefstroom, South Africa

${ }^{f}$ University of Erlangen, Erlangen, Germany

${ }^{\text {now at INFN, Padova, Italy }}$

E-mail: Christoph.Deilempi-hd.mpg.de

Collaborations managing Cherenkov telescope arrays (presently H.E.S.S., VERITAS, and MAGIC) own their data and software in private servers, only accessible to their members. However, the upcoming Cherenkov Telescope Array (CTA) will operate as an observatory, calling for powerful high-level science tools usable by the whole astronomical community.

Within the H.E.S.S. collaboration, we have produced software to export our data and instrument response functions to the standard astronomical FITS format; and to use and contribute to opensource gamma-ray astronomy data analysis packages: GammaLib/ctools, and Gammapy together with Astropy and Sherpa.

We present a summary of our experience with the FITS data formats and open source tools, comparing them to the HESS-internal data formats and tools.

The 34th International Cosmic Ray Conference,

30 July- 6 August, 2015

The Hague, The Netherlands

\footnotetext{
* Speaker.
} 